\title{
"Caminos no para llegar sino para seguir andando": una investigación poscualitativa sobre la psicología crítica en el capitalismo cognitivo
}

\author{
"Paths not to Arrive, but to Keep Walking": \\ A Post-qualitative Research on Critical Psychology \\ in Cognitive Capitalism
}

Fabio Giovanni Moreno ${ }^{1} \bowtie$, María Claudia Peralta ${ }^{2}$

${ }^{1}$ Universidad de la Sabana, Bogotá, Colombia

${ }^{2}$ Universidad de la Sabana, Bogotá, Colombia

$\square$ Campus del Puente del Común, Km. 7, Autopista Norte de Bogotá. Chía, Cundinamarca, Colombia. Correo electrónico: fabgerminal@gmail.com

Recibido: 8 de marzo del 2017 Aprobado: 27 de noviembre del 2017 Disponible en línea: 1 de abril de 2018

Cómo citar este artículo: Moreno, F. G. y Peralta, M. C. (2018). "Caminos no para llegar sino para seguir andando": una investigación poscualitativa sobre la psicología crítica en el capitalismo cognitivo. Pensando Psicología, 14(23). doi: https://doi.org/10.16925/pe.v14i23.2264

\section{Resumen}

Introducción: se planteó una investigación de tipo autorreferencial y poscualitativo con el fin de pensar la situación actual de los profesores-investigadores en la universidad, dentro del contexto global del capitalismo cognitivo y con énfasis en las posturas que han asumido un conjunto de psicólogos críticos que hubiesen sido docentes-investigadores en facultades de psicología en Colombia.

Objetivo: reconocer sentidos ambivalentes o contradictorios y plantear nuevos sentidos o sentidos emergentes acerca de la psicología crítica en Colombia, así como del rol emergente del docente-investigador en la universidad actual por medio de una orientación metodológica poscualitativa.

Método: pensar, a través de dos conceptos (diferancia y diseminación), un conjunto de autobiografías de cuatro participantes enfocadas en su desarrollo personal como psicólogos críticos y docentesinvestigadores.

Resultado: entre los materiales y la teorización realizada se encuentra que la psicología crítica se configura hoy más en una transdisciplina que en una rama o un enfoque de la psicología. La adscripción de los participantes a la psicología crítica es discontinua, es decir, cuando sucede se asocia con más fuerza a una retórica académica, a un interés más institucional y laboral que a uno puramente epistemológico o teórico. La repercusión del capitalismo académico en las facultades de psicología y en los roles actuales de la docencia y la investigación parece ser más perjudicial en términos de la calidad académica y para el bienestar de los profesores; sin embargo, parece existir también una tolerancia y un silencio acerca de estas repercusiones por parte de los docentes-investigadores.

Palabras clave: capitalismo cognitivo, investigación poscualitativa, psicología crítica. 


\title{
"Paths not to Arrive but to Keep Walking": A Post-qualitative Research on Critical Psychology in Cognitive Capitalism
}

\begin{abstract}
Introduction: Self-referential, post-qualitative research was proposed to consider the current situation of researching professors at university, within the global context of cognitive capitalism and emphasizing the stands taken by a group of critical psychologists that would have been researching professors in schools of psychology in Colombia.

Objective: To recognize ambivalent or contradictory senses and propose new meanings or emerging senses about critical psychology in Colombia, as well as the emerging role of the researching professor at current university through a post-qualitative methodology.

Method: Through two concepts (différance and dissemination), reflecting on a set of autobiographies of four participants focused on their personal development as critical psychologists and researching professors.

Result: Between the materials and the theorization conducted, we found that today critical psychology is more a transdiscipline than a branch or an approach of psychology. The participants' affiliation to critical psychology is discontinuous; that is, when it occurs, it is more strongly associated with academic rhetoric, with a more institutional and work interest than with a purely epistemological or theoretical one. The repercussion of academic capitalism both in the schools of psychology and the current roles of teaching and research seems to be more detrimental in terms of academic quality and to the well-being of professors; however, researching professors also seem to tolerate and be silent about these repercussions.
\end{abstract}

Keywords: cognitive capitalism, post-qualitative research, critical psychology.

\section{“Caminhos não para chegar, mas para seguir andando": uma pesquisa pós-qualitativa sobre a psicologia crítica no capitalismo cognitivo}

\begin{abstract}
Resumo
Introdução: propõe-se uma pesquisa de tipo autorreferencial e pós-qualitativo com o objetivo de pensar a situação atual dos professores-pesquisadores na universidade, dentro do contexto global do capitalismo cognitivo e com ênfase nas posturas assumidas por um conjunto de psicólogos críticos que teriam sido professores-pesquisadores em faculdades de psicologia na Colômbia.

Objetivo: reconhecer sentidos ambivalentes ou contraditórios e apresentar novos sentidos ou sentidos emergentes acerca da psicologia crítica na Colômbia, assim como o papel emergente do professorpesquisador na universidade atual por meio de uma orientação metodológica pós-qualitativa.

Método: pensar, através de dois conceptos (diferância e disseminação), um conjunto de autobiografias de quatro participantes enfocadas no seu desenvolvimento pessoal como psicólogos críticos e professores-pesquisadores.

Resultado: entre os materiais e a teorização, nota-se que a psicologia crítica se configura atualmente mais como uma transdiciplina do que em um ramo ou uma ênfase da psicologia. 0 vínculo dos participantes com a psicologia crítica é descontínuo, ou seja, quando ocorre, está mais fortemente associado a uma retórica acadêmica, a um interesse mais institucional e laboral, do que puramente epistemológico ou teórico. A repercussão do capitalismo acadêmico nas faculdades de psicologia e nos atuais papéis da docência e da pesquisa parece ser mais prejudicial em termos de qualidade acadêmica e para 0 bem-estar dos professores, no entanto, parece existir também uma tolerância e um silêncio sobre essas repercussões por parte dos professores-pesquisadores.
\end{abstract}

Palavras-chave: capitalismo cognitivo, pesquisa pós-qualitativa, psicologia crítica. 


\section{Introducción}

El presente artículo comenta los principales aspectos del estudio "Caminos no para llegar sino para seguir andando'. Autobiografías de psicólogos-críticos como profesores-investigadores". Se describe la situación académica al plantear el proyecto y la orientación metodológica desde la cual se desarrolló, el método y su discusión, y se destacan algunas contribuciones a la discusión sobre la definición de la psicología crítica y los psicólogos críticos, así como acerca de la situación de los profesores-investigadores en las facultades de psicología en la actualidad en el contexto global del capitalismo cognitivo. Finalmente, se consignan algunas reflexiones sobre el ejercicio de una orientación poscualitativa.

La investigación "'Caminos no para llegar sino para seguir andando'. Autobiografías de psicólogos-críticos como profesores-investigadores. Un abordaje post-cualitativo", ${ }^{1}$ se realizó como proyecto de investigación (tesis) en la Maestría en Psicología que Fabio Moreno realizó en la Facultad de Psicología de la Universidad de la Sabana. Su asesora de tesis fue María Claudia Peralta. Moreno partió de un interrogante personal: ¿Qué tenía sentido investigar a partir de su situación como estudiante de esta maestría? Aunque se debía desarrollar una temática acorde con las líneas de investigación de la maestría, Fabio Moreno le concedió prioridad a plantear un proyecto en el que pudiera aprender lo máximo de la metodología poscualitativa, así como de las reflexiones éticas actuales respecto a la investigación en ciencias sociales, y no a una problemática externa en especial. La respuesta fue pensar sus principales intereses académicos derivados de realizar en ese momento la maestría. Entrelazó tres intereses: 1. A partir de su interés en realizar la maestría como medio de acreditación para ser profesor en una universidad, una

1 El título del estudio surgió a partir de una cita realizada por Myriam Ocampo-Prado, una de las participantes, en un correo electrónico: "Diría entonces para cerrar [...] que escribir todas estas ideas producto de reflexiones teóricas o de experiencias investigativas o de acciones de aplicación de los conocimientos que se van creando en el proceso de vivir, me ha dado la oportunidad de reunir múltiples perspectivas de mi pensamiento y mi sentir, como si me mirara en un espejo de muchas caras y si bien siento que Myriam la de entonces ya no es la misma, lo que permanece sigue siendo un sentir por encima de un pensar, un caminar no para llegar sino para seguir andando como decía un poeta vallecaucano de cuyo nombre no me acuerdo. Esta frase me la enseñó el decano de Psicología de la Universidad El Bosque, gran aficionado a la lectura de poesía". línea a seguir era la situación actual de los profesores-investigadores en el contexto del capitalismo cognitivo, especialmente en el capitalismo académico; 2 . La psicología crítica como una corriente que se distingue por sus reflexiones éticas y epistemológicas acerca de las concepciones e intervenciones de la psicología en la sociedad y en la cultura, una corriente en la que se cuestiona el investigar como producción de conocimiento en y hacia los contextos sociales, así como las posiciones del investigador, y que considera la resistencia a los poderes hegemónicos (el sentido emancipatorio de las ciencias críticas) como indisociable del investigar e intervenir en la vida psicosocial; y 3. La investigación poscualitativa como una corriente crítica y divergente, especialmente frente al interpretativismo y el subjetivismo predominantes en las metodologías cualitativas actuales. Asimismo, se encontró en la investigación poscualitativa una alternativa al análisis de contenido en la que no se fragmentan y matematizan los testimonios o registros, ni se redestribuyen sus sentidos en categorías.

En el interior de la tendencia poscualitativa, Jackson y Mazzei (2012) plantean una alternativa en la investigación en ciencias sociales que consiste en "pensar-con-la teoría". Investigar consiste en problematizar los conceptos con los cuales leemos una realidad, realizar una difracción (Haraway, 1992) de los términos y conceptos con los cuales un fenómeno se nos convierte en un objeto de estudio de interés. Es decir, poner énfasis no en como los conceptos se refractan o se redicen al decir o pensar un fenómeno, sino en cómo se reinventan y se desplazan, y rastrear así las diferencias que hacen progresivamente diferencia y sus efectos (Barad, 2003; Haraway, 1992). El investigador no aspira a explicar ni a comprender una realidad sino a repensar(la) en conjunto con el material recolectado, el método y la teoría. Se trata de ir por los espacios de indiferenciación entre teoría y datos, deslizándonos de las zonas de categorización e interpretación con la intención de obtener no aquello que compruebe la teoría o las categorías naturalizadas, sino lo nuevo, lo emergente.

La idea de pensar los tres intereses señalados en un mismo estudio configuró la intención de conocer la perspectiva de los psicólogos críticos sobre los cambios actuales del orden académico y laboral del rol de profesor-investigador. Asimismo, conocer la perspectiva de psicólogos críticos que fuesen o hubiesen sido profesores-investigadores acerca de esa situación y sus implicaciones. Sin embargo, el cómo, es decir, el 
método, se le presentaba como un interés tanto o más importante que aquellas cuestiones.

Las reflexiones en las que avanzaba Fabio Moreno durante la maestría acerca de la tradición cualitativa lo llevaban a tener cautela con formulaciones subjetivizantes de la cuestión o tendientes a la develación de voces, estructuras o significados. Esta orientación coincide con las críticas planteadas por el posestructuralismo a las ideas de verdad y de sujeto, y su efecto en las concepciones ahora naturalizadas de la investigación cualitativa. Se afirma que el ser humano es un ser histórico, un ser situado en un contexto. Se afirma que la subjetividad es una condición construida socialmente, que es biopolítica, etc. No obstante, se asume que en los testimonios de los participantes reside la verdad o se pone en auge su visibilización. Como señalan Jackson y Mazzei (2012), se ha pasado de ubicar la verdad como producto de unas condiciones experimentales -como ocurre en la investigación cuantitativa-, a posicionarla en la subjetividad y en los discursos, y se tiene como método regio la interpretación en el sentido hermenéutico predominante de develación de sentidos, de esencias o de la verdad misma. ¿Hablan realmente de forma pura los participantes? Se trata de problematizar las fuerzas que están en juego en un testimonio, debido a las cuales este, aunque fuera sincero, no es "puro". Se trata de problematizar las implicaciones éticas y políticas de asumir que el investigador puede hablar por sus participantes o visibilizarlos, y puede interpretar los sentidos o significados ocultos tras sus testimonios o expresiones, o incluso comprenderlos.

Se descartó el uso de entrevistas o técnicas de recolección propiamente dialógicas con los participantes. Se contempló entonces privilegiar lo escrito sobre lo oral o hablado, así como favorecer textos autorreferenciales sobre textos con énfasis teóricos o en general "extrínsecos". Se encontró en la revisión de alternativas metodológicas que estos dilemas coincidían con el interés de Maggie MacLure (Burman y MacLure, 2005), también en la tendencia poscualitativa, en leer los textos como tales, es decir, en su naturaleza de textos y no como medios o representaciones de la mente, memoria, voces o subjetividad de los participantes. Este interés en MacLure - derivado de la influencia del pensamiento de Derrida- requería la apropiación de claves de lectura. Estas se detectaron en dos conceptos transversales en el pensamiento de Derrida: diferancia (con 'a') y diseminación. Tal y como lo afirma Adams-St. Pierre (Pearl7328, 2013), estas claves debían ser interiorizadas no solo para la lectura del material de la investigación, sino de la realidad y de mí mismo. Se trata de una táctica de divergencia epistemológica frente a lo que ella denomina "el paradigma humanista".

Incluso antes de pensar esta investigación, Fabio Moreno se encontraba inconforme con la cuestión del análisis cualitativo categorial. ¿No es acaso el análisis categorial predominante una forma de fragmentación y matematización de las transcripciones, incluso apropiada al uso de sistemas informáticos de análisis como el Atlas.ti? ¿No es una forma colonizante de investigación el leer al otro o el leer las transcripciones desde teorías o categorías preestablecidas? Enfoques como, por ejemplo, la teoría fundamentada, nos llevan más del lado de lo emergente y lo local. Sin embargo, es la noción en sí de lo categorial la que es problemática porque implica una redistribución estructural del texto, le impone esta estructura y deriva conclusiones de ella.

La divergencia analítica o interpretativa que se encuentra en Adams-St. Pierre (Pearl7328, 2013) así como en Jackson y Mazzei (2012), radica en una producción de sentidos a través de la lectura del material y no una develación de sentidos. En concreto, se trata de una serie de comentarios e interrogantes al texto (no al autor, ni a su trayectoria). Así, la autobiografía no fue en este estudio un medio para saber de la historia de vida o de la trayectoria profesional de los participantes, sino el interés material del estudio, del cual se derivan sus contribuciones.

Estas inquietudes metodológicas encontraron eco y sustentación en una tendencia a la que Elizabeth Adams-St. Pierre ha dado el nombre de "investigación post-cualitativa"2 (Adams-St. Pierre, 2011; 2014; Lather y Adams-St. Pierre, 2014; Pearl7328, 2013). Investigar, en la lógica poscualitativa, no tiene que ver con develación de verdades, voces o sentidos, ni con mecanismos de emancipación, mucho menos con una idea de acumulación de conocimiento sobre "lo mismo". Lather y Adams-St. Pierre (2014) usan el término post-cualitativa para referirse a metodologías de investigación en ciencias sociales que repiensan la ontología humanista y la epistemología característica de las metodologías cualitativas humanistas. Cuestionan conceptos tales como experiencia vivida,

\footnotetext{
2 La investigación poscualitativa sigue sin ser muy conocida en la actualidad, y en aquel momento (2014) no existían publicaciones en español acerca de ella. Es por esto que Fabio Moreno inició un blog - "Investigación poscualitativa" (Moreno, 2015) -, en el cual consigna comentarios y traducciones sobre esta corriente. Véase http://postcualitativa.blogspot.com/
} 
mundo subjetivo, lógica representacional y lógica binaria, lo cual implica poner "en borrador"3 categorías y prácticas metodológicas como, por ejemplo, problema y preguntas de investigación, revisión de la literatura, métodos de recolección de datos y análisis de datos, así como las dualidades o lógicas binarias de sí mismo/el otro, sujeto/objeto, humano/no-humano, teoría/datos. La mirada poscualitativa cuestiona la codificación y categorización de los testimonios, los diarios y las entrevistas, al considerar que son formas de fragmentación, segmentación y matematización de las fuentes, las interacciones y los sucesos de los que proviene la información a analizar y discutir. Cuestiona el privilegio de lo hablado (entrevistas, grupos focales) como fuente de la recolección de información sobre otras formas de expresión o participación (escritos, dibujos, diagramas, audios, videos, etc.) (Burman y MacLure, 2005; Jackson y Mazzei, 2012).

La investigación poscualitativa se plantea una serie de intereses de investigación que implican una lógica asubjetiva o postsubjetiva (Adams-St. Pierre, 2011; Lather y Adams-St. Pierre, 2014; Pearl7328, 2013): "Se trata de abandonar los pronombres personales (yo, tú, él, nosotros, ellos) que se refieren al sujeto humano, abandonar los nombres personales, los selves ("sí mismos"), los sujetos conocidos en los que la metodología humanista convencional está fundamentada" (Pearl7328, 2013). Se trata de una lógica posinterpretativa, es decir, una en la que se cuestionan tanto la interpretación que busca develar sentidos, verdades y esencias, como el estudio de las voces de las personas (Jackson y Mazzei, 2012). Se cuestiona la idea del análisis y discusión de los datos tendiente a "dar voz" a los participantes o a interpretar lo que hay detrás de sus respuestas o narrativas. Se llega a esta idea al pensar que lo que los participantes dicen no es "puro" implica, de hecho, una filtración y una sobreinterpretación por parte de los mismos participantes, por lo que no deben considerarse como una verdad. Los datos se consideran siempre parciales e incompletos, siempre en proceso de ser reeditados, redichos, rerecordados (Jackson y Mazzei, 2012).

Al definir la poscualitativa como orientación y, a la vez, interés de la investigación, se editó una pregunta general de investigación: ¿Cuáles sentidos ambivalentes o contradictorios y cuáles nuevos sentidos o sentidos emergentes acerca de la psicología

3 De acuerdo con el término de Derrida (1967/1971) "sous rature". crítica en Colombia y del rol emergente del docente investigador en la universidad podemos encontrar al pensar en un conjunto de autobiografías de cuatro participantes enfocadas en su desarrollo personal como psicólogos críticos y docentes-investigadores?

\section{Revisión teórica}

Se realizó una revisión teórica acerca del capitalismo académico y sus implicaciones en la vida laboral y académica de los profesores-investigadores. Se encontró que es un tema que no se ha abordado en gran medida en el contexto colombiano, a excepción del estudio de Morales (2012) en el cual se abordan las influencias del discurso gerencialista, la lógica de productividad y los efectos del capitalismo actual en las universidades, y se interesa especialmente en las prácticas de resistencia de los docentes. Sus resultados indican cómo en los profesores consultados la mayoría de las veces no existen prácticas de resistencia docente frente al capitalismo cognitivo, sino adaptación y tolerancia de las condiciones laborales emergentes, especialmente por la necesidad de conservar sus empleos:

El mundo laboral de los profesores universitarios ha sufrido en las últimas decadas una profunda transformación. Y, a juzgar por la proliferación de síntomas que la literatura vincula a la profesión docente contemporánea, parece que el cambio no está siendo para bien. A ese respecto, se observa un creciente desprestigio social del docente (al que se le responsabiliza de muchos de los males del sistema educativo), una falta de apoyo a los profesionales de la docencia desde distintos sectores y un aumento de las críticas y del cuestionamiento de su labor. A todo ello se le añaden cambios en los contenidos curriculares, nuevas exigencias de renovación pedagógica y didáctica, demandas permanentes de formación continua, cambios en las relaciones profesor-alumno, disminución de la motivación de los estudiantes para estudiar, aumento de las responsabilidades del profesorado, con la consiguiente producción de sobrecarga laboral (por la realización de múltiples y simultáneas tareas académicas, administrativas, de extensión, etc.), nuevas formas de evaluación docente centradas en las competencias y los resultados, en la eficacia y la eficiencia, flexibilización laboral y precariedad salarial, etc. (Morales, 2012, p . 221) 
En las facultades de psicología se desarrollan en la actualidad cambios de orden laboral, administrativo y cultural, así como en las subjetividades de los profesores, que han sido relacionados con el denominado capitalismo académico (Slaughter y Leslie, 1997; Slaughter y Rhoades, 2004), o en un sentido más global, con el capitalismo cognitivo (Blondeau et al., 2004).

Gómez y Celis (2007) reconocen que las transiciones y nuevas políticas académicas son expresiones del fenómeno global dentro de la sociedad de la información (Castells, 1999), especialmente en la sociedad o economía del conocimiento. Para las universidades implica una nueva orientación de sus prioridades: ingresos, patentes, regalías, poder y estatus institucional, y un mayor sentido de competitividad en relación con los indicadores de desempeño en investigación y desarrollo, así como en los ránquines de universidades. Asimismo, en la identidad de los profesores se privilegia cada vez menos la imagen o categoría del profesor-docente y más la de profesor-investigador.

Galcerán (2007) plantea que actualmente se considera conocimiento relevante aquello que pueda volverse una patente, lo que se considere necesario para el desarrollo de tareas e incluya competencias para la gestión y toma de decisiones estratégicas. La universidad pasa a ser institución que "mercadea conocimiento", de manera que la prioridad es la "productividad académica", mientras la docencia pierde poco a poco reconocimiento y estatus dentro y fuera de ella. Los sistemas de remuneración y reconocimiento se reconfiguran para favorecer a los profesores-investigadores más productivos en términos de papers, artículos, libros y contratos. Esto configura lo que algunos han denominado "capitalismo cognitivo", y su expresión más específica asociada a las universidades: el capitalismo académico.

Alvesson (2012) comenta algunas señales de estos fenómenos en la vida de los investigadores académicos. Plantea, por ejemplo, el uso del término ROI-search, a fin de contrastarlo con el término tradicional RE-search (“investigación"). La RE-search está asociada a la curiosidad, al juego incertidumbre-conocimiento y a las contribuciones sociales que puede brindar un proyecto de investigación. Por el contrario, la ROI-search se refiere a la investigación basada en criterios de retorno de la inversión (return on investment, ROI), no solo en los retornos económicos, sino en las expectativas que tienen los investigadores de ser reconocidos a través de una publicación en una revista de alto impacto, así como los premios y recompensas en términos salariales y de estatus. La ROI-search, por tanto, se caracteriza por su énfasis en los productos instrumentales.

De acuerdo con Alvesson (2012), para el investigador actual se vuelven muy importantes el carrerismo, el narcisismo, la propia subjetividad y los signos de éxito, los cuales deben ser bien visibles: ¿dónde has publicado?, ¿cuántas citas tienes en Google? De ahí que la tendencia sea a encasillarse en temas para dominarlos bien y a partir de ellos desarrollar la mayor productividad.

Alvesson (2012) advierte sobre algunas actitudes actuales en los académicos que, si bien son legítimas, limitan la creación y la comunicación de conocimiento relevante y significativo: 1 . El interés polarizado en la actualización de sí mismos o en lo que es importante para ellos; 2 . El hedonismo o idea de optimizar el factor de placer en la investigación y en la publicación; 3 . El interés en demostrar la propia competencia y valor como persona capaz de publicar un artículo en una revista importante; 4. La importancia dada a ciertas clases de discurso y al uso de lenguaje complicado e inaccesible; y 5 . El ideal de pertenecer y satisfacer a una red particular de investigadores.

Estas transformaciones políticas, económicas y académicas repercuten en las instituciones universitarias de Colombia, bien sea por vía de competitividad o bien por vía de los requerimientos estatales. Así, por ejemplo, se encuentran el Decreto 1279 de 2002 sobre el régimen salarial y de prestaciones de los docentes en universidades públicas, las agendas del Ministerio de Educación sobre la educación superior, las políticas de Colciencias, etc., todo lo cual viene implementándose con fuerza a fin de asimilar y acoger las visiones, los intereses y las prioridades propias del capitalismo cognitivo (Galcerán, 2007; Gómez y Celis, 2007).

Se realizó también una revisión sobre la corriente de la psicología crítica (Parker, 1999; 2009, Montero, 2010), con algunas especificidades en el contexto colombiano. Fueron especialmente importantes la revisión histórica realizada por Nelson Molina y Ángela María Estrada (Molina-Valencia y Estrada Mesa, 2006), y la entrevista a Teresa Cabruja realizada por Camilo Pulido (Pulido-Martínez, CarvajalMarín y Cabruja-Ubach, 2009) de la cual proviene la expresión "el sinuoso fluir de la psicología crítica".

Proporcionar una definición de la psicología crítica es una cuestión compleja, y es uno de sus temas de reflexión el cómo hacerlo, así como las implicaciones éticas y epistemológicas que tiene dar una u 
otra definición. Es necesario problematizar la adopción que se realiza en Latinoamérica de las definiciones y planteamientos de la teoría crítica y la psicología crítica provenientes del extranjero. Estos procesos también podrían contrastarse con un enfoque poscolonial. Teresa Cabruja señala que no solo cada país, sino cada comunidad, debe construir su psicología crítica (Pulido y Cols, 2009).

Los textos revisados (Parker 1999, 2009; MolinaValencia y Estrada-Mesa, 2006; Montero, 2010; Pulido-Martínez et al., 2009) destacan algunos temas propios de la psicología crítica: la construcción social de la realidad, los fenómenos de la ideología o la comunicación y la conciencia; interaccionismo simbólico, etnometodología, estudio de cultura e ideología, activación social y exclusión como problema psicosocial. Asimismo, la cuestión de la participación y el compromiso, la intersubjetividad y la cuestión del poder son recurrentes en estos autores.

También se abordan temas conceptuales como, por ejemplo, identidad. Interesa como fenómeno psicosocial, pero también importa el concepto situado en una ideología, en las ciencias sociales (Montero, 2010). La psicología crítica es reflexiva de sus fundamentos, es decir, estos son tema de interés y la reflexividad misma también (Molina-Valencia y Estrada-Mesa, 2006). Otro tema de la psicología crítica tiene que ver con las implicaciones políticas, sociales y culturales de las investigaciones y lo que informan, por ejemplo, al privilegiar unos temas e invisibilizar otros (Parker, 2009).

Los temas que aborda la psicología crítica también se encuentran asociados a las trayectorias de los investigadores, como puede observarse en la selección de temas que plantea Ian Parker (1999, 2009): resistencia y poder, psicología y cultura, psicología y cotidianidad. Teresa Cabruja, en cambio, resume del siguiente modo su trayectoria en la psicología crítica:

Comentaré que empecé con la construcción socio-histórica-cultural de la locura desde la antipsiquiatría y las aportaciones de Foucault, y pasé, más en general, al interés por la intersubjetividad y las relaciones de poder (gobernabilidad y resistencia). Mezclé la perspectiva feminista postestructuralista y la crítica étnica-cultural postmodernista, con la crítica a la Psicología. (Pulido et al., 2009, p. 3)

Este comentario contrasta con los índices y esquemas ordenados que pretende presentarnos Parker y nos abre un tema interesante a problematizar en futuras discusiones y estudios dentro de la cuestión de la institucionalización de la psicología crítica: la estética y las metáforas de los discursos sobre la psicología crítica.

Un aspecto distintivo de la institucionalización de la psicología crítica en Colombia es que surge en el contexto de la psicología social, la cual a su vez puede considerarse, en general, un antecedente de la psicología crítica, pero en Colombia fue particularmente determinante, tal como lo indican Nelson Molina y Ángela María Estrada (2006). Esto ha generado debates conceptuales acerca de si la psicología crítica siempre es social. La revisión teórica realizada aquí nos lleva a la conclusión de que no necesariamente lo es, ya que existen diversos temas que la psicología social no se plantea y la psicología crítica sí, especialmente la autorreferencia y los fundamentos (MolinaValencia y Estrada-Mesa, 2006) en los que sobresale la teoría crítica, entre otros (Parker, 1999). Asimismo, podríamos decir -en los términos que plantea Habermas (1990) en Conocimiento e Interés-, que la psicología crítica tiene la naturaleza de una ciencia emancipatoria, mientras que la psicología social en general tiene diversas agendas, entre las que existe una psicología social crítica.

\section{Diseminación y diferancia (con 'a')}

Se trata de los dos conceptos que fueron interiorizados por Fabio Moreno como claves de lectura y de divergencia epistemológica frente a la inercia del paradigma humanista (Pearl7328, 2013), es decir, el paradigma subjetivista e interpretativista. La diferancia (con a), en francés différance, es una palabra inventada por Derrida (1967/1971) para referirse a una propiedad de los significados en la escritura: remiten siempre a otros significados ad infinitum. Es decir, no solo se diferencian de otros, sino que su sentido siempre es en referencia a otros significados $y$, por tanto, el significado de un término no es estable o determinado, sino que siempre se pospone, está en movimiento y permanece oculto o en reserva. El término différance juega con los términos en francés para diferencia (differénce) y diferir (différer), los cuales se usan tanto para los sentidos de "diferenciar" y "distinguir", como para los de "posponer" y "remitir". Solo en la escritura de la palabra différance se puede ver la diferencia con la palabra differénce, ya que se leen de la misma forma, con lo cual Derrida también quiere destacar y resistir al discurso hegemónico y opresor (o lenguaje oral) sobre la 
escritura. Derrida (1989) señala que no existe un centro primordial de significado, y cómo en toda postulación de un centro opera un ocultamiento de dicho vacío.

Uno de los sentidos de la expresión diseminación (Derrida 2007) está asociado a la polisemia, esto es, toda expresión puede ser prolífica en sentidos y apropiaciones que incluso pueden resultar contradictorios entre sí, tal como ocurre con la expresión pharma$\cos$, la cual en griego se puede usar como "medicamento" y como "veneno". Sin embargo, de una forma más profunda, la exposición que sobre la diseminación hace Derrida (2007) nos remite a otros sentidos como la crítica a la dualidad habla/escritura y a la tradicional hegemonía del habla que Derrida rastrea a partir de Platón. Más aún, a partir de otros comentarios sobre el concepto de mimesis en relación con el de diseminación, Derrida plantea cómo la significación (el sentido) implica siempre disyuntivas y pliegues.

\section{Método}

Este trabajo se realizó desde la perspectiva poscualitativa y con el uso de autobiografías de cuatro psicólogos críticos. La autobiografía puede entenderse como la técnica de recolección de información.

Los cuatro participantes fueron Myriam Ocampo-Prado, ${ }^{4}$ Álvaro Díaz-Gómez ${ }^{5}$, Nelson Molina-Valencia ${ }^{6}$ y Bruno Jaraba-Barrios. ${ }^{7}$ El criterio de selección de los participantes fue el siguiente:

\footnotetext{
4 Myriam Ocampo-Prado. Psicóloga egresada de la Universidad del Valle. Doctora en Psicología de la Escuela de Altos Estudios en Ciencias Sociales de París. Docente-investigadora y profesora visitante en varias universidades. Directora y consultora de programas en políticas públicas en el área de desarrollo comunitario y protección de los derechos humanos. Ha trabajdo en los campos de psicología social, psicología política y psicología ambiental.

5 Álvaro Díaz-Gómez. Psicólogo egresado de la Universidad Incca (Bogotá). Magíster en Filosofía, en Psicología Comunitaria, y en Educación. Candidato a Doctor en Ciencias Sociales en el Cinde (Manizales) y en la Universidad de Salamanca. Profesor asociado de la Universidad Tecnológica de Pereira. Actualmente es el coordinador del Nodo de Psicología Social Crítica de Ascofapsi.

6 Nelson Molina-Valencia. Psicólogo de la Universidad de los Andes (Bogotá). Magíster y Doctor en Psicología Social de la Universidad Autónoma de Barcelona. Miembro del Taos Institute. Fundador de la Asociación Ibero Latinoamericana de Psicología Política. Profesor asistente y subdirector de Investigaciones y Postgrados del Instituto de Psicología de la Universidad del Valle.

Bruno Jaraba-Barrios. Psicólogo de la Universidad del Norte (Barranquilla) y Magíster en Sociología de la Universidad Nacional. Ha publicado artículos de investigación sobre la historia crítica de la psicología en Colombia y sobre sociología de la psicología. Profesor instructor en la Pontificia Universidad Javeriana.
}

debían ser o haber sido recientemente profesores de planta en una universidad en Colombia, y para ese momento haber desarrollado proyectos de investigación desde la psicología crítica. Debían tener reconocimiento disciplinar en la psicología crítica (por artículos publicados y afiliaciones). Fueron seleccionados de manera intencional y con un criterio de ejemplaridad.

Ellos accedieron a escribir una autobiografía enfocada, principalmente, en su desarrollo personal como profesores-investigadores y como investigadores suscritos a la psicología crítica. En la autobiografía también debían definir o expresar cómo entendían el ser investigador desde la corriente crítica y de qué manera definían o entendían el ser o haber sido profesores-investigadores o docentes-investigadores en una facultad en Colombia. A estos textos se sumó una carta de cierre con comentarios finales que también quedó incluida en el material analizado. El uso de esta técnica no replicó algún uso previo realizado en otro estudio o desde algún autor en particular. El análisis consistió en una serie de comentarios sobre los textos distribuidos en las siguientes claves o núcleos: 1 . Sentidos ambivalentes o contradictorios; 2 . Nuevos sentidos o sentidos emergentes; y 3. Interrogantes a pensar.

\section{Discusión}

Los participantes, al escribir su autobiografía, así como al tener una entrevista de trabajo, al hablar a sus estudiantes en clase o al hacerlo fuera de las aulas se ubican en unas coordenadas, están en distintas posiciones y juegos del lenguaje, coordenadas dentro de zonas de conversación que se generan en cada circunstancia (Davies y Harré, 1999). Esta idea nos permite pensar en la ambigüedad o ambivalencia en el definirse como "psicólogo crítico" evidenciada en el análisis de los cuatro materiales. En algunos momentos, dentro de una misma autobiografía es apropiado hacerlo, mas en otros pasajes puede haber una crítica a esa clase de definiciones y se prefiere hablar de una identidad interdisciplinar o de investigador social, etc. ¿Ante quién necesitan o buscan los psicólogos críticos definirse como tales? ¿Cuándo no es apropiado hacerlo? Es una exploración que también podría extenderse a los personajes que comienzan a configurarse como íconos en el caso de la psicología crítica, como es el caso de Ian Parker, o aun en el contexto latinoamericano de la psicología social crítica con Maritza Montero. Es decir, si bien existen unos 
argumentos teóricos en esas identidades o adscripciones, este interrogante emergente se refiere más a pensar en las funciones retóricas de tales enunciados.

En los cuatro materiales la psicología crítica se trata de otra disciplina o metadisciplina, quizás transdisciplina, que se materializó o necesitó históricamente tomar la imagen de una psicología, o de una rama de la psicología (la "psicología crítica"). Un ejemplo:

Un psicólogo crítico no debería llevar ese nombre, sino más bien el de crítico de la psicología, pues no toma a esta como su medio de conocimiento ni como referente de identidad profesional, sino como objeto de análisis. Un psicólogo crítico se acerca a la psicología con estrategias de todo tipo menos psicológicas. No es de extrañar que invariablemente concluya que lo psicológico no es psicológico. Pero ese es su trabajo: despsicologizar el mundo. (Autobiografía de Bruno Jaraba)

Como lo advierten Pulido-Martínez et al. (2009) en su artículo, que lleva el sugestivo título "El sinuoso fluir de la psicología crítica", los discursos latinoamericanos de la psicología crítica o social crítica tienden a no pensar su propia psicología crítica, y a importar programas de investigación e intereses de otras partes.

La definición de una psicología crítica es aún problemática y esto se evidencia en algunos de los materiales analizados en este estudio, en los que esta se observa como un estudio social vestido al modo de la psicología, o nombrado así quizá también con una serie de funciones retóricas de reconocimiento dentro del campo disciplinar de la psicología o del campo institucional de la psicología, o de las ciencias sociales (por ejemplo, las facultades). En conjunción con esto, un aspecto común de los cuatro materiales es la no adscripción a escuelas o a autores, aunque cada uno tiene su manera particular de manifestarlo (no obstante, en los cuatro casos se hizo explícito).

El término "crítico" o "crítica" de la psicología crítica es en todos los materiales uno de esos términos que Derrida (1989) llamaría "centro-vacío": un término que, siendo clave e incluso el núcleo de un discurso, no se define, y cuya naturalización implica un ocultamiento de cómo dicho centro no existe, o de cómo para existir remite a otros sentidos.

Los cuatro materiales autobiográficos tuvieron en común ignorar la cuestión del ser profesor-investigador en la actualidad y lo que esto implica en el rol laboral. A través de unas cartas suplementarias se tuvo la oportunidad de obtener algunos comentarios al respecto, pero estos se realizaron más desde una perspectiva teórica y no subjetiva, a excepción quizás de algunos comentarios sobre disonancias teóricas con las facultades. Los comentarios son diversos. Así, se encuentra en una de las cartas una perspectiva tolerante y que justifica estos cambios asociados al capitalismo cognitivo dentro de un enfoque de la calidad universitaria, mientras que en otra se afirma que la mezcla de docencia e investigación — sin dar una mayor independencia y peso a algunas de estas misiones - resulta perjudicial tanto para bienestar del profesor como para su tarea. Tanto en la carta de Nelson Molina como en la de Bruno Jaraba se expresa un desacuerdo en relación con que el criterio de evaluación de las publicaciones de un profesor-investigador sea el número o la cantidad de publicaciones, y no el tipo y la calidad, y de qué manera esto no solo tiene que ver con las políticas de las facultades, sino con las políticas de Colciencias. También coinciden en señalar que los modos de producción y evaluación académica no son favorables para la consolidación de la comunidad científica nacional, así como tampoco para producir conocimiento relevante dirigido a la sociedad, lo cual coincide con los análisis de Galcerán (2007) y Morales (2012).

En este sentido, uno de los textos de Myriam Ocampo señala:

En las facultades de psicología se vive una situación semejante a la del campo aplicado, las relaciones autoritarias y en búsqueda de conservar la hegemonía priman sobre las relaciones democráticas y favorables al ejercicio de la libertad. Hacer de la cátedra un espacio para la construcción de conocimiento dialógico es muchas veces motivo de envidias y competencia entre docentes y entre estos y las directivas. Con gran frecuencia prima no la constitución de comunidades de saber o de pensamiento o de círculos para construir teorías y nuevos aportes a la comprensión de la realidad sino el desgaste de trabajar por la docencia y a veces la investigación cuidando de no despertar la sospecha que se está granjeando el apoyo de los estudiantes y generando gérmenes de nuevas hegemonías. El docente investigador debe hacer gala de una capacidad de adaptación y de realizar múltiples concesiones en aras de conservar su posición. Este ambiente conspirativo no permite organizar comunidades de saber como una condición propia de la actividad académica, son esfuerzos individuales los 
que logran aparecer en el escenario de esta competencia. El mayor capital existente es el capital del conocimiento y de la creación, lograr desarrollarlo y ser reconocido por ello es una tarea, yo diría de suerte, implica encontrar interlocutores que no se sientan cuestionados en sus propios territorios y se sientan atraídos a incluir los planteamientos ajenos. Por eso es una cuestión de "suerte", de "ángel". Las facultades de psicología en Colombia no escapan a este juego, los directivos sensibles a dejar pensar a sus subordinados procuran condiciones para que la creación, la propuesta de nuevas preguntas, el desarrollo de aportes innovadores o promotores de cambios, se materialice en acciones y transformaciones. (Carta de cierre, Myriam Ocampo)

En síntesis, puede decirse que dentro de los materiales y la teorización realizada se encuentra que la psicología crítica se está configurando más en una transdisciplina que en una rama o enfoque de la psicología. La adscripción por parte de los participantes a la psicología crítica es discontinua: cuando sucede se asocia con más fuerza a una retórica académica, a un interés más institucional y laboral, que a uno puramente epistemológico o teórico.

Si bien la repercusión del capitalismo académico en las facultades de psicología y en los roles actuales de la docencia y la investigación parece ser más perjudicial en términos de la calidad académica y en el bienestar de los profesores, parece existir también una tolerancia y un silencio acerca de estas repercusiones por parte de los docentes-investigadores.

La adopción de una perspectiva poscualitativa también implicó algunas incompatibilidades dentro de la facultad, particularmente dentro del comité de investigación que supervisa la evolución del trabajo de investigación, y desde el cual también fue un punto de discusión encontrar lectores afines o apropiados para el paradigma en el que se piensa y desarrolla el estudio. Según los participantes, adoptar una perspectiva poscualitativa no fue un tema de especial interés.

Así, por ejemplo, en el caso de Fabio Moreno como autor, escritor y lector implicó una dificultad recurrente que denominó "inercia epistemológica" del pensamiento interpretativista y subjetivista, no solo en lo académico o teórico, sino también en lo personal, pues la cotidianidad está especialmente conformada para pensarnos a nosotros mismos y a los demás como individuos y personas. De igual forma, a buscar lo común, la comparación y la generalización.
Dado lo anterior, insistimos en que aquí solo derivamos conclusiones en torno a una serie de textos y estamos lejos de hablar por "la psicología crítica" o de caracterizar a "los psicólogos críticos", ni siquiera aspiramos a hablar de las personas de los participantes; y, como se señaló en la introducción, en la revisión teórica y en el análisis no se busca visibilizar sus voces ni sus subjetividades.

De esta manera, no se asumió una orientación antisubjetiva ni antiinterpretativa. Más bien, como se ha descrito en la revisión, sería una crítica dessubjetivista y desinterpetativista. En este sentido, los conceptos o los discursos sobre subjetividad, sujeto psicológico o sí mismo no son núcleos temáticos de este abordaje.

La autobiografía es un tema entendido de manera convencional y predominante como un medio de reflexión sobre lo subjetivo y el sí mismo del autor, etc. (Arfuch, 2014; Wang y Brockmeier, 2002). A fin de leer las autobiografías de la forma que se desarrolló en la investigación, esas preguntas no fueron necesarias $y$, de hecho, se excluyeron, ya que el interés radicaba en lo que se denominaría "la lógica del texto", que en cada autobiografía es incontrastable a la de cualquier otra, incluidas las demás presentadas y analizadas en este estudio.

La perspectiva crítica poscualitativa nos lleva a pensar en la subjetividad como un macrodiscurso contemporáneo, una supracategoría dentro de las ciencias sociales. Es un discurso que, pese a su auge, la mayoría de las veces no piensa acerca de su sentido y justificación, ni acerca de ser eso: discurso. Como tal, pertenece no solo a una situación histórica y a un enclave social y cultural correspondiente a quien o quienes la enuncien, sino también a juegos de verdad y a retóricas en las que aparece naturalizado o sin problematizar.

Cuando se pensó en las autobiografías no se tenía el objetivo o un interés especial en la reconstrucción de una trayectoria en sí, sino en cómo escribían o "se escribía" esa convergencia del trabajo investigativo desde la psicología crítica y el trabajo académico o rol laboral de profesor-investigador.

En general, frente a las inercias epistemológicas internas y las objeciones o no correspondencias externas se realizó el ejercicio que se nombró como "resistencia epistemológica". Una resistencia que continúa la resistencia del paradigma cualitativo frente a las presiones de la tradición y la hegemonía del pensamiento positivista, pero también es una resistencia frente al paradigma cualitativo y de lo que "debe 
ser" un estudio cualitativo, en general, de lo que "es" ciencia. Se trata también de una resistencia epistemológica frente a la inercia epistemológica en nosotros mismos como investigadores y lectores de los textos, así como de la inercia del pensamiento - en nosotros mismos o en los textos-que pone como centro al sujeto, a la verdad, a las voces, a las esencias o a la experiencia de sí.

\section{Referencias}

Adams-St. Pierre, E. (2011). Post Qualitative Research: The Critique and the Coming After. En N. Denzin y Y. Lincoln (Edit.), The Sage handbook of qualitative research (pp. 611-625). Thousand Oaks, California: Sage

Adams-St. Pierre, E. (2014). A Brief and Personal History of Post Qualitative Research. Toward "Post Inquiry". Journal of Curriculum Theorizing, 30(2), 2-19. Recuperado de https://goo.gl/azedhP

Alvesson, M. (2012) Do We Have Something to Say? From Re-search to Roi-search and Back Again. Organization 20(1) 79-90. doi: 10.1177/1350508412460996

Arfuch, L. (2014, marzo). (Auto)biografía, memoria e historia. Clepsidra. Revista Interdisciplinaria de Estudios sobre Memoria, 1, 68-81.

Barad, K. (2003). Posthumanist Performativity: Toward an Understanding of How Matter Comes Matter. Signs: Journal of Women in Culture and Society, 28(3), 801831.

Blondeau, O., Dyer-Whiteford, N., Vercellone, C., Kyrou, A., Corsani, A., Rullani, E. Moullier Boutang, Y. y Lazzarato, M. (2004) Capitalismo cognitivo, propiedad intelecual y creación colectiva. Madrid: Traficantes de Sueños.

Burman, E., y MacLure, M. (2005) Deconstruction as a Method of Research. B. Somekh y C Lewin (Eds.), Research Methods in the Social Sciences (pp. 284-292). Londres: Sage.

Castells, M. (1999). La era de la información. Economía sociedad y cultura. La sociedad red (Vol. 1). México: Siglo XXI.

Davies, B. y Harré, R. (1999, enero-abril). Posicionamiento: la producción discursiva de la identidad. Sociológica, 14(39), 215-239.

Decreto 1279 de Junio 19 de 2002. Por el cual se establece el regimen salarial y prestacional de los docentes de las Universidades Estatales. Junio 19 de 2002. Bogotá: Congreso de la República de Colombia.
Derrida, J. (1971). De la gramatología. México: Siglo xxi.

Derrida, J. (1989). La estructura, el signo y el juego en el discurso de las ciencias humanas. En La escritura y la diferencia (pp. 383-401). Barcelona: Anthropos.

Derrida, J. (2007). La diseminación. Madrid: Fundamentos/ Espiral.

Galcerán, M. (2007). Reflexiones sobre la reforma de la universidad en el capitalismo cognitivo. Nómadas, 27, 86-97.

Gómez, V. M., y Celis, J. E. (2007). Docencia, estatus, distinción y remuneración. Nómadas, 27, 98-109.

Habermas, J. (1990). Conocimiento e interés. Madrid: Taurus.

Haraway, D. (1992). The Promises of Monsters: A Regenerative Politics for Inappropriate/d Others. En L. Grossberg, C. Nelson y P. Treichler (Eds.), Cultural studies (pp. 295-337). Nueva York: Routledge.

Jackson, A. Y. y Mazzei, L. A. (2012) Thinking with Theory in Qualitative Research. Viewing Data across Multiple Perspectives. Londres: Routledge.

Lather, P., y Adams-St. Pierre, E. (2014) Post-qualitative Research. International Journal of Qualitative Studies in Education, 26(6), 629-633. doi: 10.1080/ 09518398.2013.788752

Molina-Valencia, N., y Estrada-Mesa, A. M. (2006) Critical Construction of Psychology in Colombia. Annual Review of Critical Psychology, 5, 342-353.

Montero, M. (2010, julio-diciembre). Crítica, autocrítica y construcción de teoría en la psicología social latinoamericana. Revista Colombiana de Psicología, 19(2), 2010, 177-191.

Morales, M. (2012). Prácticas de resistencia docente en la universidad orientada al mercado. Capturas y fugas académicas en el contexto colombiano (investigación doctoral). Barcelona: Universitat Autònoma de Barcelona.

Moreno, F. G. (2015). Investigación post-cualitativa [Blog]. Recuperado de http://postcualitativa.blogspot.com/

Parker, I. (1999) Critical Psychology: Critical Links. Annual Review of Critical Psychology, 1, 3-18.

Parker, I. (2009) Psicología crítica: ¿Qué es y qué no es? Revista Venezolana de Psicología Clínica Comunitaria 8, 139-159.

Pearl7328. (2013, julio 26). Post qualitative research: the critique and the coming after. Conferencia de Elizabeth Adams-St. Pierre [Archivo de video]. Recuperado de https://www.youtube.com/watch?v=7mbbVHD_kyM 
Pulido-Martínez, H., Carvajal-Marín, L. M. y Cabruja-Ubach, T. (2009). El sinuoso fluir de la psicología crítica: una conversación con Teresa Cabruja Ubach. Universitas Psychologica, 8(3), 583-599. Recuperado de https://goo.gl/QvzzwS

Slaughter, S. y Leslie, L. (1997). Academic Capitalism: Politics, Policies and Entrepreneurial University. Baltimore: Johns Hopkins University Press.
Slaughter S. y Rhoades, G. (2004). Academic Capitalism and New Economy: Markets, State and Higher Education. Baltimore: Johns Hopkins University Press.

Wang, Q.y Brockmeier, J. (2002). Autobiographical Remembering as Cultural Practice: Understanding the Interplay Between Memory, Self and Culture. Culture \& Psychology, 8(1), 45-64. doi: 10.1177/1354067X020 